and drug related problems (DRPs) from medicines reviews (MRs); and benefits for patients and healthcare professionals (HCPs).

Materials and Methods The report builds mainly on studies, mini-audits and questionnaires. Four master thesis/projects completed in 2012 in our region studied the IMM model in hospital and primary care. Two mini-audits were completed during 2012 as benchmarking of daily activities and recording of MEs. Three questionnaire surveys were conducted; one investigating clinical pharmacists' experiences with the model, the second exploring the attitudes of and usefulness for HCPs and the third was a patient satisfaction survey.

Results Up to $70 \%$ of patients had one or more discrepancies between the drug lists in hospital and at home. Most discrepancies were due to drug(s) missing in the drug history. On average 2.1 DRPs per patient were identified and acted upon. Most DRPs were classified as: need for additional treatment and choice of drug/dose not appropriate. HCPs and pharmacists rated the service highly (5.1-5.5 on a 6-point scale) with regard to patient benefits and usefulness for HCPs.

Conclusions The model has been successfully implemented in hospitals in Central Norway. Further research will be needed to investigate end points such as reduced length of hospital stay and time to readmission. We plan to provide a more extensive service to all patients in our region, also in the community.

No conflict of interest.

\section{CPC-052 EVALUATION OF THE INTEGRATION OF A CLINICAL PHARMACIST WITHIN A MOBILE MULTIDISCIPLINARY GERIATRIC TEAM}

doi:10.1136/ejhpharm-2013-000276.509

${ }^{1}$ A Passanisi, ${ }^{2} \mathrm{~A}$ Coenen. ${ }^{1}$ Centre Hospitalier du Bois de l'Abbaye et Hesbaye, Pharmacy, Seraing, Belgium; ' ${ }^{2}$ Centre Hospitalier du Bois de l'Abbaye et Hesbaye, Geriatric Unit, Seraing, Belgium

Background In any general hospital, the number of elderly patients admitted in wards other than geriatric wards is steadily rising. The 'Centre Hospitalier du Bois de l'Abbaye et de Hesbaye' gets the benefit from a mobile second-line multidisciplinary team whose mission is to contribute to provide medical specialists and their staff with general geriatric principles and multidisciplinary expertise. The inclusion of a clinical pharmacist in this multidisciplinary team is an effective way to optimise the quality and the efficacy of elderly patient health care.

Purpose To evaluate the impact of including a clinical pharmacist within the mobile multidisciplinary geriatric team on the efficacy of pharmaceutical care.

Materials and Methods Two different working methods of the clinical pharmacist were compared in order to evaluate her inclusion in the geriatric team.

The first method, used from 1 July to 31 December 2011, evaluated the treatments and the interventions provided by the clinical pharmacist.

The second method, used from 1 January to 30 June 2012, was identical to the first one except that the interventions provided by the clinical pharmacist were taking into account the observations made by the multidisciplinary team.

Results From 1 July to 31 December 2011, 187 interventions were made for a total of 78 elderly patients. From 1 January to 31 May 2012, 202 interventions were made for a total of 75 elderly patients.

Following the inclusion of the clinical pharmacist within the multidisciplinary team we observed an improvement in the efficacy of pharmaceutical care with an increase of $12 \%$ in the number of interventions.

Conclusions The inclusion of a pharmacist within the mobile multidisciplinary geriatric team enables him/her to make better use of his/her expertise and to improve his/her analysis, improving patient health care.

No conflict of interest.

\section{CPC-053 EVALUATION OF THE MANAGEMENT OF DIABETIC FOOT IN RABTA NATIONAL TEACHING HOSPITAL}

doi:10.1136/ejhpharm-2013-000276.510

'M Razgallah Khrouf, ${ }^{2} \mathrm{M}$ Turki, ${ }^{3} \mathrm{~A}$ Louhaichi, ${ }^{3} \mathrm{M}$ Guerfali. 'Hospital the "Rabta", Pharmacy, Tunis, Tunisia; 'Departement of Pharmacology, Pharmacology, Monastir, Tunisia; ${ }^{3}$ Hopital La RABTA, Pharmacy, Tunis, Tunisia

Background Feet lesions are the greatest cause of diabetic consultations in the endocrinology service.

Purpose To evaluate the diabetic foot management in a Tunisian hospital in order to improve patients' quality of life.

Materials and Methods This was a prospective, descriptive study based on documentation regarding 43 cases from the endocrinology service at Rabta hospital over five months. Data collected included: the age of the patient, sex ratio, type of diabetes, duration and type of lesion. The diagnostic examinations selected were: Doppler exploration, standard radiography of the foot, bacteriological sample of pus (applied to 2 patients). The prescribed treatment and the evolutionary aspects were also documented.

Results In our study we present 43 diabetics with foot lesions. Sex ratio (men/women $=3.3$ ), median age 60 years and median length of diabetes 15 years. Traumatic lesions represented $46.68 \%$. The most frequent lesions were gangrene (32.55\%), ulcer and painful perforating plantar ulcers $(67.45 \%)$. The main aetiological factors were peripheral neuropathy $(72.09 \%)$ and arthritis of the lower limb $(30.23 \%)$. Osteitis and diffuse atheromatous infiltration were observed in $46.66 \%$ of the patients. Samples were taken from two patients. $90.70 \%$ of the patients benefited from antibiotic treatment, the most prescribed drugs were amoxicillin + ac. clav $(30 \%)$, fusidic acid (22\%), pristinamycin (22\%) and ciprofloxacin (15\%). An amputation was performed on $37.20 \%$ of the patients

Conclusions Sepsis of the diabetic foot remains one of the most severe complications in Tunisia; it represents a frequent reason for prescribing antibiotics. This encourages strict microbiological investigation to identify the causative germs and the need for perfect observance of the rules of antibiotic prescription.

No conflict of interest.

\section{CPC-054 EVALUATION OF THE USE OF CAPSAICIN PATCHES IN GARCIA DE ORTA HOSPITAL}

doi:10.1136/ejhpharm-2013-000276.511

P Tavares Almeida, A Alcobia. Hospital Garcia de Orta, Pharmacy, Almada, Portugal

Background Capsaicin is commonly used in creams in low concentrations with limited success. More recently it has been formulated in a high concentration patch $(8 \%)$, indicated for the treatment of peripheral neuropathic pain in non-diabetic adults.

Purpose To evaluate the effectiveness of treatment with capsaicin patches in a group of patients in Garcia de Orta Hospital Pain Unit.

Materials and Methods This retrospective study, which included 30 patients with neuropathic pain, examined data from the last two years. This treatment was done more than once, with a minimum interval of 12 weeks.

The number of treatments and the number of patches, the area affected, the perception of pain, functional capacity and adverse events were evaluated.

Results The mean age was $58.1 \pm 16.5$, the number of treatments was $2.8 \pm 0.7$, the number of patches per treatment was $2.4 \pm 1.0$ with an average cost per treatment of $€ 630.2 \pm € 262.6$. 